\title{
ANÁLISE DAS HOSPITALIZAÇÕES POR USO DE DROGAS ENTRE CRIANÇAS E ADOLESCENTES NO ESTADO DO PARANÁ, BRASIL
}

\section{Alexandre Dido Balbinot}

Mestrando do Programa de Pós-Graduação em Saúde Coletiva pela Universidade do Vale do Rio dos Sinos (UNISINOS), São Leopoldo, RS, Brasil. E-mail:

\section{Arieli Haubert}

Educadora Social; Pedagoga pela Cesuca da Faculdade Inedi, Cachoeirinha, RS, Brasil.
RESUMO: Este estudo teve como objetivo analisar a evolução das hospitalizações psiquiátricas de crianças e adolescentes por uso de substâncias psicoativas no sistema público de saúde e o tempo médio de permanência no Estado do Paraná, utilizando-se como indicadores as taxas de internações entre os anos de 2000 a 2015. Trata-se de um estudo ecológico que utilizou dados secundários provenientes do Sistema DATASUS. A análise foi composta por Regressão de Poisson com variância robusta e Correlação de Spearman. O nível de significância utilizado foi de 5\%. Observou-se aumento em 5\% nas taxas de hospitalizações, com aumento de $4 \%$ para o sexo masculino e $8 \%$ para o feminino. Houve aumento no tempo de duração das hospitalizações em $6 \%$, sendo de $4 \%$ para o sexo masculino e $9 \%$ para o feminino. Evidenciou-se correlação positiva entre o tempo médio e as taxas de internações, permanecendo o fenômeno quando estratificado por sexos. Então, apesar das políticas da reforma psiquiátrica, os serviços substitutivos não estão suprindo em sua totalidade a demanda de crianças e adolescentes em situação de drogadição.

PALAVRAS-CHAVE: Hospitalização; Saúde Mental; Sistema Único de Saúde.

\section{HOSPITALIZATION DUE TO DRUGS AMONG CHILDREN AND ADOLESCENTS IN THE STATE OF PARANÁ, BRAZIL}

\begin{abstract}
The evolution of psychiatric hospitalizations of children and adolescents due to psychoactive substances in the public health system is analyzed. Current investigation also deals with mean permanence in the state of Paraná, Brazil, by hospitalization rates between 2000 and 2015 as indicators. Current ecological study employed secondary data retrieved from the DATASUS database. Analysis comprised Poisson regression with robust variance and Spearman's Correlation at 5\% significance level. A 5\% increase in hospitalization rates was reported, a $4 \%$ increase for males and an $8 \%$ increase for females. Duration period of hospitalizations increased by $6 \%$, with $4 \%$ for males and $9 \%$ for females. There was a relative correlation between mean time and hospitalization rates, with the same phenomenon for gender. In spite of policies on the psychiatric reform, substitute services do not totally supply demand of children and adolescents in conditions of drug addiction.
\end{abstract}

KEY WORDS: Hospitalization; Mental Health; National Health System. 


\section{INTRODUÇÃO}

O consumo de substâncias psicoativas não é um fenômeno recente na sociedade, mas permeia as diferentes sociedades ao longo do tempo e tem se modificado. Na atualidade o consumo é considerado um relevante problema de saúde pública, com repercussão direta em diferentes áreas da vida do sujeito que realiza este uso. Mais que isto, sabe-se que quanto mais precoce a experimentação, maior a probabilidade da ocorrência de dados decorrentes deste uso (REIS; UCHIMURA; OLIVEIRA, 2013; CARDOSO; MALBERGIER, 2014).

A preocupação dos diferentes seguimentos da sociedade frente à população de usuários permeia cada vez idades mais precoces. Este fato é decorrente da ampliação da precocidade da experimentação de diferentes substâncias, tanto álcool e tabaco, quando outras drogas ilícitas. Segundo o II Levantamento do CEBRID, dos sujeitos com 12 a 17 anos da Região Sul, $58,8 \%$ relatam uso na vida de álcool e $21,3 \%$ de tabaco, sendo que o uso de outras drogas acomete pelo menos 7,0\% da população de mesma idade. Observa-se, assim como em outros estudos, elevadas prevalências e com aumento ao longo do tempo (CARLINI et al., 2006; CARLINI et al., 2010).

Deste modo, observa-se que as estratégias de prevenção devem contemplar não somente adolescentes, mas também crianças. Independentemente da questão da faixa etária abordada para programas de prevenção, devese ter em mente que a legislação brasileira referente aos jovens, ao vetar a venda e a disponibilização de substâncias psicoativas para os mesmos, sendo o descumprimento passível de penalização legal, conforme disposto no ECA (Estatuto da Criança e do Adolescente), artigo 243 e Lei Complementar $\mathrm{n}^{\circ}$ 13.106/15 (BRASIL,1990).

Para compreender os prejuízos ocasionados pelo uso de substâncias psicoativas na infância e na adolescência é preciso definir quais períodos são esses no ciclo vital. Cronologicamente, é considerado criança o sujeito que está entre 0 e 12 anos. Já a adolescência se inicia nesta idade até os 18 anos, ainda que haja discussões onde neurocientistas afirmem que a adolescência iria até os 21 anos em função de que apenas nesta idade o cérebro humano atingiria a maturidade completa (CARDOSO;
MALBERGIER, 2014; ALMEIDA FILHO; FERREIRA; GOMES; SILVA; SANTOS, 2007; SOARES, 2010; OLIVEIRA et al., 2009).

Assim, os prejuízos nesses sujeitos são potencializados justamente pela imaturidade neuronal e de todos os outros órgãos: quanto mais jovens, maiores as lesões causadas nestes organismos, sofrendo também, porém, a interferência do padrão de uso. Além disso, é necessária a reflexão quanto aos aspectos psicológicos da infância e da adolescência, podendo-se caracterizar a primeira pelo quanto são influenciáveis estes sujeitos, experimentando em função da oferta. Já os adolescentes muitas vezes experimentam no intuito de inserir-se em um grupo que faz uso. A dependência em ambos os casos muitas vezes ocorre em função da busca pelas sensações trazidas por tais substâncias (CARDOSO; MALBERGIER, 2014; ALMEIDA FILHO; FERREIRA; GOMES; SILVA; SANTOS, 2007; SOARES, 2010; OLIVEIRA et al., 2009).

A rede de saúde pública brasileira, através do Sistema Único de Saúde (SUS), vem compreendendo essa problemática e buscando criar estratégias de prevenção e tratamento. Em consonância com a reforma psiquiátrica, são disponibilizados para tratamento além dos leitos psiquiátricos em hospitais, o atendimento de crianças e adolescentes pode ser feito através do CAPSi (Centro de Atenção Psicossocial Infantojuvenil) ou conjuntamente com adultos usuários de drogas nos CAPSad (Centro de Atenção Psicossocial - Álcool e Drogas) (COELHO et al., 2014).

Porém, também há estratégias de redução de demanda, buscando a prevenção; este é o trabalho das equipes do Programa Estratégia de Saúde da Família (ESF). Estas equipes, através das UBS (Unidades Básicas de Saúde), cada uma em um território, buscam reduzir a experimentação através da conscientização e, em alguns casos, onde a experimentação já ocorreu, tentam com que esta não se torne dependência (PEREIRA, 2013; FORTESKI; RADUENZ; SACHETTI, 2013; BRASIL, 2002).

Deste modo, o presente estudo teve como objetivo analisar a evolução das hospitalizações psiquiátricas de crianças e adolescentes por uso de substâncias psicoativas no sistema público de saúde e o tempo médio de permanência no Estado do Paraná, utilizando-se como indicadores as taxas de internações entre os anos de 2000 a 2015. 


\section{MÉTODO}

Trata-se de um estudo ecológico que analisa as hospitalizações de crianças e adolescentes a partir das taxas de internações, assim como o tempo médio de permanência dos sujeitos nas respectivas hospitalizações decorrentes de transtornos mentais e comportamentais por uso de álcool e outras substâncias psicoativas, em ambos os sexos, em idade igual ou inferior a 19 anos, tendo ocorrido as mesmas no Estado do Paraná, no período entre 2000 a 2015.

Os dados foram acessados através do Sistema de Informações de Saúde, disponibilizado pelo Departamento de Informática do SUS - DATASUS. Mais especificamente, foram acessados os dados sobre "Informações de saúde", na aba "Epidemiológica e morbidade" e no grupo "Morbidade hospitalar do SUS". A coleta dos dados ocorreu no mês de março de 2016.

Os dados referentes ao tamanho populacional foram acessados também nas "Informações de Saúde" do DATASUS, porém, na aba "Demográficas e socioeconômicas", na opção "Projeção da população das unidades da federação por sexo e grupos de idade: 20002013".

Para a análise foram constituídas as seguintes variáveis dependentes:

Taxa de hospitalizações por diagnósticos de Transtornos Mentais e comportamentais devido ao uso de álcool e outras substâncias psicoativas (F10 - F19);

Tempo médio de permanência hospitalizado por diagnósticos de Transtornos Mentais e comportamentais devido ao uso de álcool e outras substâncias psicoativas (F10 - F19).

Para a construção das variáveis relacionadas às taxas foi utilizada a seguinte fórmula: (Valor das hospitalizações por grupo de causas por sexo no ano) / (Tamanho da população por sexo no ano) x 100.000 habitantes.

Após a coleta dos dados, os mesmos foram inseridos em planilhas no Programa Microsoft Excel $^{\circledR} \mathrm{e}$ posteriormente transferidos através do Programa Stat Transfer $9^{\circledR}$ para o Programa STATA $11.1^{\circledR}$ onde realizouse a análise dos dados.

A análise dos dados foi composta por Regressão de Poisson com variância robusta e Correlação de Spearman. Foi empregado Intervalo de Confiança de 95\% e nível de significância de 5\%.

O estudo faz uso de dados secundários, ou seja, de domínio público. As informações utilizadas são disponibilizadas pelo Ministério da Saúde a toda população através do sistema de informações DataSUS, deste modo não se identificando a necessidade de submissão do estudo para avaliação por comitê de ética em pesquisa.

\section{RESULTADOS}

Nesta análise foram abarcadas 19.123 internações hospitalares ao longo do período estudado, sendo 14.392 referentes ao sexo masculino e 4.731 ao feminino. Foram em média 1.195,19 (DP: 571,13; 657-2.444) hospitalizações por ano. O sexo masculino apresentou média de 899,5 (DP: 348,76; 552-1.657) hospitalizações ao ano, enquanto o sexo feminino 295,69 (DP: 224,51; 105-787) hospitalizações ao ano.

Mais especificamente, referente às taxas de internações totais, observou-se média de 1.77 (DP: 0,87; 0,97-3,67) hospitalizações por 100.000 habitantes na faixa etária abordada ao ano, sendo que a menor taxa ocorreu no ano de 2009 e a maior no ano de 2011. Para o sexo masculino, evidenciou-se média de 2,61 (DP: $1,05 ; 1,60-4,88)$ hospitalizações por 100.000 habitantes ao ano. O menor valor ocorreu no ano de 2009 e o maior no ano de 2011. As evoluções dos coeficientes de hospitalizações psiquiátricas por sexo são apresentadas abaixo, no Gráfico 1. 


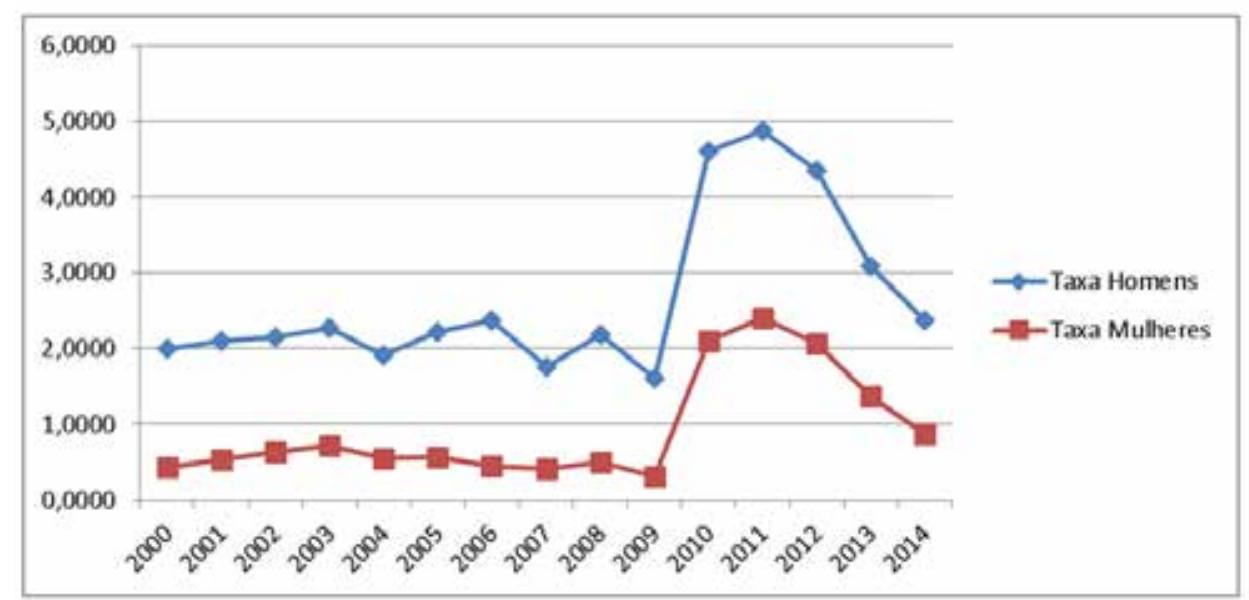

Gráfico 1. Coeficientes de hospitalizações psiquiátricas devido aos transtornos mentais e comportamentais por uso de álcool e outras substâncias psicoativas por crianças e adolescentes de acordo com sexo, Paraná, 2000-2015

O tempo médio das internações foi, em média, de 23,49 (DP: 8,42; 13,6-37,8) dias por hospitalização, tendo ocorrido o menor valor no ano de 2006 e o maior nos anos de 2011 e 2013. Para o sexo masculino, o tempo médio foi de 21,82 (DP: 5,77; 13,6-32,2) dias por hospitalização, ocorrendo o menor valor no ano de 2006 e o maior valor no ano de 2013. Já o sexo feminino apresentou tempo médio de hospitalização de 28,91 (DP: 19,$99 ; 14-70,8$ ) dias por hospitalização, tendo ocorrido o menor valor no ano de 2006 e o maior no ano de 2011. As evoluções dos tempos médios de permanência nas hospitalizações psiquiátricas por sexo são apresentadas abaixo, no Gráfico 2.

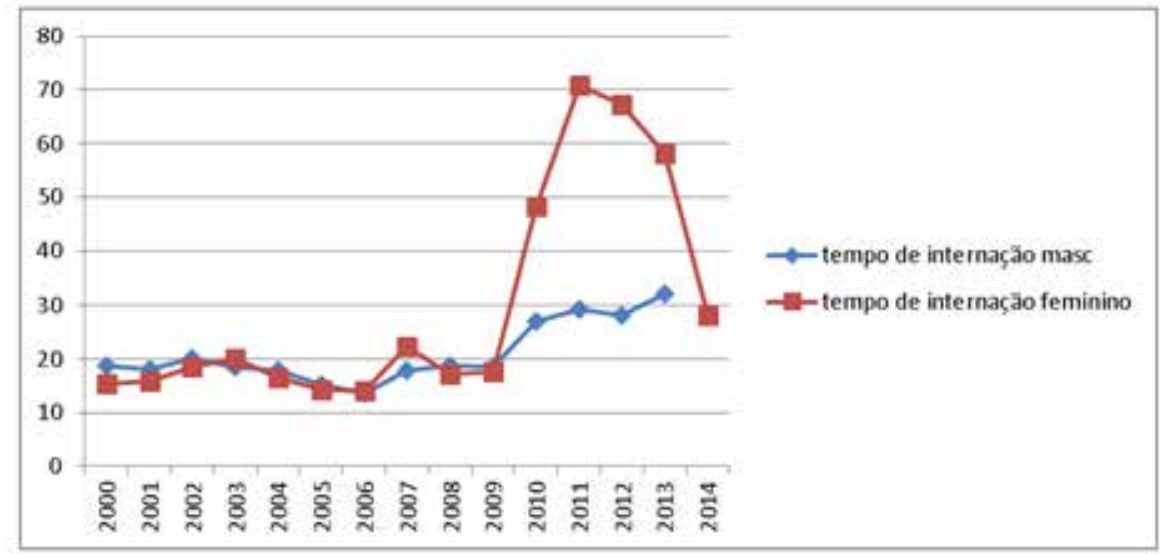

Gráfico 2. Evolução do tempo médio das hospitalizações psiquiátricas devido aos transtornos mentais e comportamentais por uso de álcool e outras substâncias psicoativas por crianças e adolescentes de acordo com sexo, Paraná, 2000-2015

Ao longo do período estudado, foi observado aumento significativo em 5\% ( $p<0,05)$ nas taxas de hospitalizações, semelhante ao evidenciado para os diferentes sexos, com aumento de $4 \%(\mathrm{p}<0,05)$ para $o$ sexo masculino e de $8 \%(\mathrm{p}<0,05)$ para o sexo feminino.

Também foi observado aumento significativo no tempo de duração das hospitalizações sendo que este aumento foi em torno de $6 \%(\mathrm{p}<0,05)$. Em relação aos grupos sexuais, houve aumento de $4 \%(\mathrm{p}<0,05)$ para o sexo masculino e $9 \%(\mathrm{p}<0,05)$ para o feminino.

A descrição completa da análise das taxas de hospitalizações psiquiátricas e tempo de permanência são apresentadas abaixo, na Tabela 1. 
Tabela 1. Regressão de Poisson para taxas de internações e tempo médio de duração das hospitalizações psiquiátricas devido aos transtornos mentais e comportamentais por uso de álcool e outras substâncias psicoativas por crianças e adolescentes de acordo com sexo, Paraná, 2000-2015

\begin{tabular}{lcccc}
\hline Variável dependente & Sexo & $\begin{array}{c}\text { Coeficiente de } \\
\text { Regressão }\end{array}$ & IC 95 \% & $\boldsymbol{p}$-valor \\
\hline Taxas de hospitalizações & Masculino & 1,04 & 1,00 a 1,07 & $0,029^{*}$ \\
& Feminino & 1,08 & 1,02 a 1,14 & $0,011^{*}$ \\
\hline Tempo médio de duração das hospitalizações & Total & 1,05 & 1,00 a 1,09 & $0,021^{*}$ \\
\hline & Masculino & 1,04 & 1,03 a 1,06 & $<0,001^{*}$ \\
& Feminino & 1,09 & 1,03 a 1,15 & $0,002^{*}$ \\
\hline & Total & 1,06 & 1,03 a 1,08 & $<0,001^{*}$ \\
\hline
\end{tabular}

$* p<0,05$

Através da análise de Correlação de Spearman foi possível evidenciar correlação positiva entre o tempo médio das hospitalizações e as taxas de internações tanto nos valores para o total da amostra quanto para os diferentes grupos quando estratificado pelo sexo dos sujeitos.
A descrição completa da análise das correlações entre as taxas de hospitalizações psiquiátricas e o tempo de permanência são apresentadas abaixo, na Tabela 2 .

Tabela 2. Correlação de Spearman para taxas de internações e tempo médio de duração das hospitalizações psiquiátricas devido aos transtornos mentais e comportamentais por uso de álcool e outras substâncias psicoativas por crianças e adolescentes de acordo com sexo, Paraná, 2000-2015

\begin{tabular}{lccc}
\hline & Sexo & Coeficiente de Regressão & $\boldsymbol{p}$-valor \\
\hline Transtornos por uso de Álcool e outras Drogas & Masculino & 0,499 & $0,049^{*}$ \\
& Feminino & 0,665 & $0,005^{*}$ \\
\hline & Total & 0,599 & $0,014^{*}$ \\
\hline
\end{tabular}

*p $<0,05$

\section{DISCUSSÃO}

Mesmo com o advento da reforma psiquiátrica e a constituição de serviços substitutivos às hospitalizações psiquiátricas, permanece a tendência de utilização do atendimento através das internações psiquiátricas. Pelo menos, no âmbito do atendimento à drogadição. Essa evolução vai em contramão ao proposto pela reforma psiquiátrica, e, mais que isto, vai ao encontro do movimento contra-reforma, movimento este que propõe a continuidade da disponibilização e até mesmo ampliação dos atendimentos através de leitos hospitalares.

O aumento das taxas de internação hospitalar demonstra um rearranjo político e administrativo que propicia a ampliação na capacidade de atendimento disponibilizada para a população da faixa etária estudada. Ainda, reflete a preocupação e o clamor da sociedade civil para o desenvolvimento de respostas imediatistas no atendimento da drogadição. Entretanto, a hospitalização psiquiátrica é também uma amarração do sujeito a uma forte estigmatização (SCISLESKI; MARASCHIN; SILVA, 2008).

Parece ainda ser o reflexo dos elevados patamares de experimentação e consumo de substâncias psicoativas galgados pela população de jovens, não restringindo-se mais apenas ao álcool e tabaco, mas estendendo-se às substâncias que apresentam como característica a maior facilidade de dependência e propensão ao surgimento de 
agravos, havendo um consumo de drogas extremamente elevado principalmente entre jovens em situação de rua (GALDURÓZ, 2001).

A internação de jovens para desintoxicação é, sem dúvida, em muitos casos, uma etapa fundamental para a aquisição e manutenção da abstinência do consumo de drogas. É, em primeiro lugar, uma medida de proteção que garante o início da abstinência, que talvez não conseguisse ser alcançada com o sujeito estando em seu ambiente de origem.

A manutenção da abstinência nos primeiros dias também é favorecida no ambiente hospitalar, isto se apoia no conhecimento de que o pico de craving (fissura) durante a abstinência se concentra no período de até duas semanas. Então, o período inicial da abstinência aparece como um momento de fragilidade onde o sujeito pode desenvolver um lapso ou até mesmo uma recaída.

É interessante ainda pensar o papel das hospitalizações por ordem judicial, que estão em crescimento, dentro deste processo de modificação das taxas dentro do período estudado, pois como discutido no trabalho de Scisleski, Maraschin e Silva (2008), pode ser um fator de auxílio ou de facilitação do imediatismo da aquisição de vagas em leitos hospitalares. Todavia, também tem sido utilizada como uma forma de punição aos jovens ou suas famílias, e deixa de ter o caráter de exceção (DE SOUZA; TEIXEIRA; GONÇALVES, 2014).

Mais que isto, o processo jurídico e os diferentes campos de saber envolvidos no desenrolar da internação compulsória reduzem os responsáveis e o papel familiar a algo considerado impotente, o que é incorporado por aqueles sujeitos dali em diante. Entretanto, o aparato social do Estado não consegue dar conta daquilo que é o papel da família e que, por se sentir impotente, está deixando de fazer; é produzida, em muitos casos, a desproteção do sujeito (DOS REIS; DE FATIMA GUARESCHI; DE CARVALHO, 2015).

Uma hipótese elencada previamente para o aumento das taxas de internação foi o tempo de duração das mesmas. Ou seja, quanto menor o tempo de internação há, consequentemente, maior vacância do leito e a possibilidade de uma maior rotatividade. Entretanto, este fato não foi evidenciado na presente análise, pois os aumentos das taxas correlacionaram-se positivamente com o tempo de duração das internações.

Os resultados podem refletir o fenômeno descrito em estudo de Coelho et al. (2014), que aponta a modificação do perfil de atendimento dos leitos hospitalares. É apontada uma inversão nas patologias que ocasionaram a internação, sendo atualmente maior o contingente de hospitalizações decorrentes da experimentação e consumo de substâncias psicoativas. Assim, é plausível pensar que o aumento das internações observadas no presente estudo esteja associado à modificação do perfil de atendimento.

É evidente ainda a diferença no contingente das taxas de internação entre os sexos. Os achados do presente estudo corroboram com outros estudos, como de Alves e Kossobudzky (2002), realizado em Curitiba, os quais demonstraram que o perfil das internações hospitalares para tratamentos de uso de drogas entre jovens é caracterizado pelo maior contingente proveniente do sexo masculino. Este fenômeno parece decorrer do maior uso de drogas entre o sexo masculino e aponta para outros estudos que apresentaram menor número de internações para o sexo feminino (FILHOA et al., 2003; BORINI; GUIMARÃES; BORINI, 2003).

Por fim, é importante lembrar que qualquer modalidade de atendimento disponibilizada e efetivada para o sujeito é melhor do que a ausência de atendimento. Também é necessário que sejam avaliadas as possiblidades de atendimento alternativas à internação hospitalar, buscando-se a efetividade do tratamento para os sujeitos em uso abusivo e dependentes. Porém, é importante acima de tudo, que sejam implementadas políticas e ações de prevenção à experimentação e consumo já em idades precoces, assim como estratégias para minimização dos agravos (SCIVOLETTO; HENRIQUE JR; ANDRADE, 1996).

\section{CONCLUSÃO}

Através deste estudo foi possível evidenciar que apesar das políticas da reforma psiquiátrica, os serviços substitutivos não estão suprindo em sua totalidade a demanda de crianças e adolescentes em situação de drogadição. Além disso, políticas de prevenção também não estão sendo suficientemente efetivas a ponto 
de reduzir a experimentação precoce das variadas substâncias.

Sendo assim, sugere-se estudos complementares que abranjam a forma de prevenção realizada, bem como a importância da rede de apoio familiar e escolar nesta prevenção da precocidade e conscientização dos danos ocasionados pelas diversas substâncias, a fim de aprimorar estes serviços e alcançar com maior eficiência os objetivos.

\section{REFERÊNCIAS}

ALVES, R.; KOSSOBUDZKY, L. A. Caracterização dos adolescentes internados por álcool e outras drogas na cidade de Curitiba. Interação em Psicologia, v. 6, n. 1, 2002.

ALMEIDA FILHO, A. J.; FERREIRA, M. A.; GOMES, M. L. B.; SILVA, R. C.; SANTOS, T. C. F. O adolescente e as drogas: consequências para a saúde. Esc Anna Nery Rev Enferm., v. 11, p. 605-610, 2007.

BRASIL. Lei n. 8.069, de 13 de julho de 1990. Dispõe sobre o Estatuto da Criança e do Adolescente e dá outras providências. Diário Oficial da União, Brasília, 16 de julho de 1990

BRASIL. Portaria/GM do Ministério da Saúde no 336-02, de 19 de fevereiro de 2002. Estabelece CAPSI, CAPS II, CAPS III, CAPSi II e CAPS ad II. Brasília, DF, 2002.

BORINI, P.; GUIMARÃES, R. C.; BORINI, S. B. Usuários de drogasilícitasinternados em hospital psiquiátrico: padrões de uso e aspectos demográficos e epidemiológicos. J Bras Psiquiatr, v. 52, n. 3, p. 171-9, 2003.

CARDOSO, L. R. D.; MALBERGIER, A. Problemas escolares e o consumo de álcool e outras drogas entre adolescentes. Psicol. Esc. Educ., Maringá, v. 18, n. 1, p. 27-34, Jun. 2014.

CARLINI, E. A. et al. II Levantamento domiciliar sobre o uso de drogas psicotrópicas no Brasil: estudo envolvendo as 108 maiores cidades do país. São Paulo: CEBRID - Centro Brasileiro de Informação sobre Drogas Psicotrópicas; UNIFESP, 2006.
CARLINI, E. A. et al. VI Levantamento nacional sobre o consumo de drogas psicotrópicas entre estudantes do ensino fundamental e médio das redes públicas e privadas de ensino nas 27 capitais brasileiras - 2010 . São Paulo: CEBRID - Centro Brasileiro de Informações sobre Drogas Psicotrópicas, 2010.

COELHO, V. A. A. et al. Alteração do perfil de atendimento dos hospitais psiquiátricos públicos de Belo Horizonte, Brasil, no contexto da reforma da assistência à saúde mental. Ciênc. saúde coletiva, v. 19, n. 8, p. 1305-16, 2014.

DE SOUZA, L. A. F.; TEIXEIRA, J. D.; GONÇALVES, R. T. Meninas confinadas. Perfil das jovens em cumprimento de medida de internação em São Paulo e no Pará. Rev Bras Adolescência e Conflitualidade, n. 10, 2015.

DOS REIS, C.; DE FÁTIMA GUARESCHI, N. M.; DE CARVALHO, S. Discursos sobre Família e Risco nas Internações Compulsórias de Usuários de Drogas. Psicologia, v. 46, n. 3, p. 386-399, 2015.

FERREIRA FILHO, O. F.; TURCHI, M. D.; LARANJEIRA, R.; CASTELO, A. Perfil sociodemográfico e de padrões de uso entre dependentes de cocaína hospitalizados. Rev Saúde Pública, v. 37, n. 6, p. 751-9, 2003.

FORTESKI, R.; RADUENZ, M.; SACHETTI, V. A. R. Relato de Experiência: Procedimento de sala de espera em um CAPSAD. R. Saúde públ. Santa Cat., Florianópolis, v. 6, n. 3, p. 120-133, jul./set. 2013.

GALDURÓZ, J. C. F. Uso e abuso de drogas psicotrópicas no Brasil. Revista IMESC, v. 3, p. 37-42, 2001.

OLIVEIRA, L. N. et al. Vulnerabilities of children admitted to a pediatric inpatient care unit. Rev. paul. pediatr., São Paulo, v. 32, n. 4, p. 367-373, dez. 2014.

PEREIRA, E. D. Assistência à saúde mental na atenção primária à saúde. 2013. 34f. Trabalho de conclusão de curso (Especialização em saúde pública) - Universidade Federal do Rio Grande do Sul, Porto Alegre: 2013.

SCISLESKI, A. C. C.; MARASCHN, C.; SILVA, R. A. N. Manicômio em circuito: os percursos dos jovens e a internação psiquiátrica. Cad. Saúde Pública, v. 24, n. 2, p. 342-352, 2008. 
SCIVOLETTO, S.; HENRIQUE JR., S. G.; ANDRADE, A. G. Proposta de tratamento para adolescentes usuários de drogas - A internação domiciliar como alternativa. Rev Neuropsiquiatria da infância e adolescência, v. 4 , n. 1, p. 33-40, 1996.

SOARES, H. L. R.; GONCALVES, H. C. B.; WERNER JUNIOR, J. Cérebro e o uso de drogas na infância e adolescência. Fractal, Rev. Psicol., Rio de Janeiro, v. 22, n. 3, p. 639, dez. 2010.

Recebido: 29 de março de 2016

Revisado: 01 de abril de 2016

Aceito: 14 de abril de 2016 